\title{
Bioelectronic Medicine and the Dawn of Robotic Training to Improve Motor Outcome in Chronic Stroke
}

\author{
Bruce T Volpe $e^{1,2}$ \\ ${ }^{1}$ The Feinstein Institute for Medical Research, Manhasset, New York, United States of America; ${ }^{2}$ Hofstra North Shore-LIJ School of \\ Medicine, Hempstead, New York, United States of America
}

\begin{abstract}
Engineers and clinicians have cooperated to produce and test new classes of bioelectronics that have altered motor impairment that occurs after stroke. The rationale that increased intensity of training alters outcome derives from past clinical and preclinical work. Now several studies have demonstrated that interactive robotic devices are a potent tool for the therapist to deliver effortlessly, reproducible high intensity movement training. These robots are safe and can provide a platform so that recovery might be influenced by a combination of noninvasive novel treatment programs. Also, these robotic devices provide a continuous objective history of movement parameters that will open the horizon for their use in generating novel movement biomarkers to understand, predict and measure the influence of new treatments on motor outcome after neurological injury.
\end{abstract}

Online address: www.bioelecmed.org

doi: 10.15424/bioelectronmed.2014.00002

\section{INTRODUCTION}

The enormous personal and societal burden caused by diseases of the brain and spinal cord make imperative innovative attempts to reduce illness and alter permanent disability. Colleagues at MIT, HI Krebs and N Hogan, developed an array of interactive robotic devices that we have used to aid and abet treatment programs for neurological recovery of motor function of the limbs in patients who have had a stroke (1-4). These interactive robots move a patient's paretic arm and when the patient begins to move, these robots "get out of the way" so the patient can execute the movement with very little resistance from the device. With these robots, a therapist can generate training sessions that are intensity controlled (a single one-hour session requires over 1,000 to and fro move- ments of a limb segment). The robots are tireless agents that produce reliable, reproducible movement sequences. In addition, the controllers on these robotic devices can be tuned to individual patients so that the robot can present different physical challenges at the point when a patient is moving the robot arm, so that training can focus, for example, on the speed, trajectory (aiming) or force of a movement $(5,6)$. The success of controlled multicenter randomized studies that used robotic protocols to improve the outcome of upper limb motor recovery in patients with chronic stroke prompted the American Heart Association to make robotic training standard care (5,7-9). This work will review the continued relationship between rehabilitation robotics for the paretic upper extremity after chronic stroke and focus on

Address correspondence to Bruce T Volpe, The Feinstein Institute for Medical Research, 350 Community Drive, Manhasset, NY 11030. Phone: 516-562-3384; Fax: 516-562-2953;

E-mail: Bvolpe1@nshs.edu.

Submitted June 23, 2014; Accepted for publication September 9, 2014; Published Online (www.bioelecmed.org) December 2, 2014.

\section{The Feinstein Institute for Medical Research} Empowering Imagination. Pioneering Discovery.

frequent questions that arise in clinical practice: Can intensive training alter the dreaded "performance plateau"? Can the human-robot interaction be optimized for an individual patient? Can explicit training of the affected limb generalize to improvement on untrained motor tasks? Can the control group be trained in an intensive manner to mimic the robot training? Can the robot-derived measurements of movement provide an objective biomarker for studies of other treatments, especially new pharmacology for acute stroke? Can the robot training emerge as a platform for combination therapy?

\section{CAN INTENSIVE ROBOTIC TRAINING ALTER A PERFORMANCE PLATEAU?}

Standard treatment for sensorimotor impairment after stroke must focus on the unaffected limbs to teach the patient to adapt and compensate, especially with motor abilities that mediate eating, grooming, bathing and attending to bathroom functions. The learning situation that characterizes an inpatient stay at an acute stroke rehabilitation facility is fraught not only with the stress of adjusting to new life circumstances, but knowing that the moment one stops improving, discharge is imminent. This failure to re- 
$1 \mathrm{~A}$

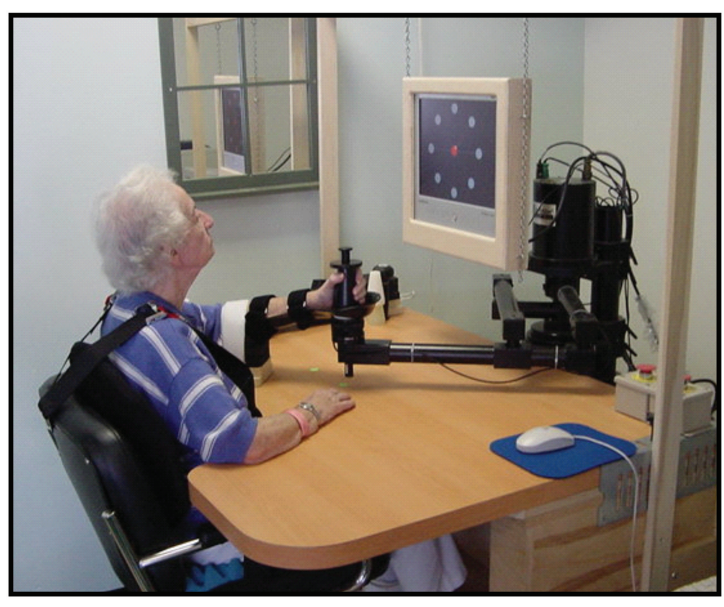

1B

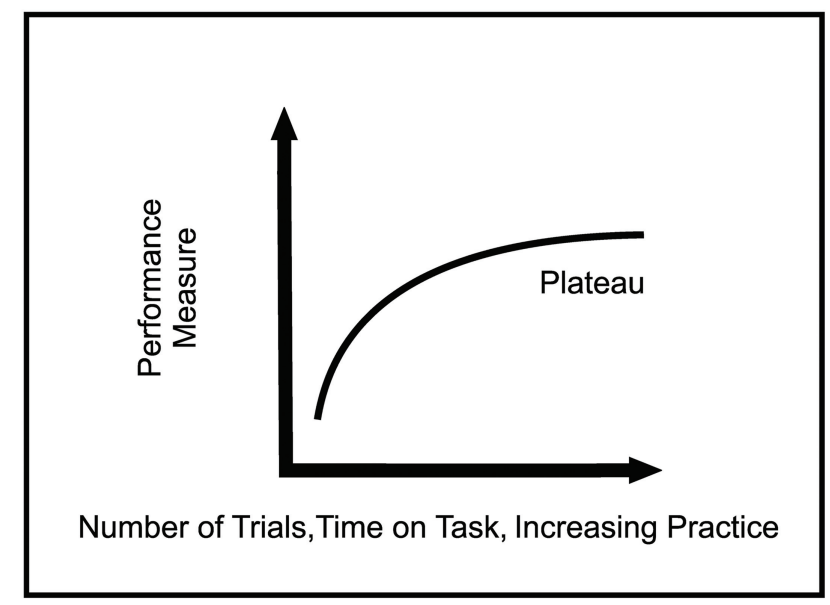

Figure 1. (A) This panel illustrates a patient sitting in a chair in front of the robotic device. Her arm is fastened to a trough and she is grasping the handle of the robot. She is comfortably strapped into the chair so that movement of the robot can be accomplished only by moving her shoulder and elbow. This position and control of the patient's limb illustrates a key feature of the training; the patient is required to focus on moving only the affected limb. The patient looks at a video screen which displays the position of her hand and the end of the robot which is a flashing active point that traces her executed movements. (B) The panel indicates typical performance measure plateaus as trials or time on task or practice increase. Panel A is adapted from (4): Bruce T Volpe, Patricio T Huerta, Johanna L Zipse, Avrielle Rykman, Dylan Edwards, Laura Dipietro, Neville Hogan, Hermano I Krebs. Robotic Devices as Therapeutic and Diagnostic Tools for Stroke Recovery. Arch. Neurol. 2009;66:1086-90. See http://archneur.jamanetwork.com/article.aspx?articleid=797921.

spond positively to a program is termed a "plateau" and is incorrectly considered an optimal motor outcome (10). In fact, a plateau may indicate a consolidation period and not a diminished capacity for motor improvement (11). For example, Figure 1 could well represent the effect of repeated or daily episodes of physical therapy for a patient recovering from limb weakness caused by stroke, and the measure of any particular performance would plateau. The rate of improvement tends toward zero as practice continues, or, phrased differently, the same practice has a diminishing effect on performance. These patients would be scheduled for discharge from training programs.

But in a comparable group of patients with chronic stroke, all of whom had been discharged from inpatient and outpatient programs because they had reached a "plateau," the performance data after novel robot training reveal different results. In this experiment, 248 consecutive patients with chronic stroke underwent robotic treatment for upper extremity weakness, employing 6-wk training sessions (1-h sessions three times a week) $(4,12)$. The $x$ axis in Figure 2 reflects the individual scores on a well-known clinical motor measurement scale, the Fugl-Meyer (range 0, severe impairment to 66, normal upper extremity function) (13), and the black line indicates the admission scores of patients with a wide range of stroke severity. The $y$ axis reflects cumulative probability (a normalization scheme to view large data groups) and the inset illustrates the movement of this group of patients from admission to midtraining to discharge and follow-up, which occurs 3 months after therapy has finished.

The movement of the curves rightward toward higher Fugl-Meyer values is significant (Kolmogorov-Smirnoff, $p<$ 0.001). The details are instructive and show that for patients with severe impairment and scores in the 20s, the improvement occurred in the first nine treatments, yet for those with moderate impairment and scores in the 30s, the improvement occurred throughout the training. Moreover, for some with this moderate impairment, there was further improvement after the training ceased. That a performance plateau should be considered reaching an optimum or having diminished capacity for improvement should give way to more refined individualized and varied treatment approaches.

\section{CAN THE HUMAN-ROBOT INTERACTION BE OPTIMIZED FOR AN INDIVIDUAL PATIENT?}

These particular robots guide and move the patient's arm, and when the patient begins to move, the motors cease, although there is a constant record of the speed profile so the measurement capacity of the robotic device is not affected. Recently the robot controllers have been programmed so that a particular movement can be resisted, or the aiming of a movement can be guided, or the speed of the movement can be damped or enhanced $(6,14)$. In the next experiment, patients with chronic stroke $(n=111)$ underwent 9 wks of every other day training 


\section{$2 \mathrm{~A}$}

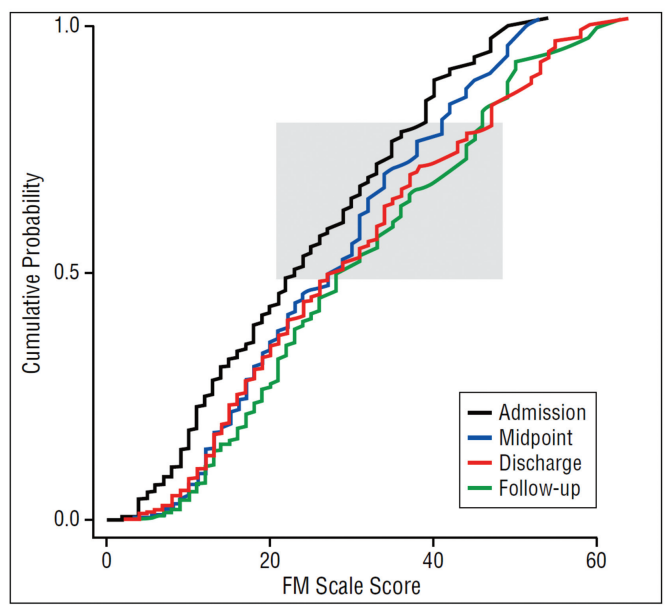

Figure 2. (A) This panel shows the range of stroke severity (measured by Fugl-Meyer (F-M or FM) scale score) in patients with chronic stroke $(n=248$; mean age $=62.3$ years, range 17-89; $5 \mathrm{~d}$ to 11.3 years after stroke) on admission (black line), at midpoint in treatment (blue line), at discharge (red line) and at follow up (green line) 3 months after robot training ceased. Treatment consisted of 1-h robotic sessions 3 d per week for 6 wks. The plot of values moves significantly to the right over time and training and indicates higher F-M scale scores at the subsequent evaluation times (Kolmogorov-Smirnoff, $p<0.001$ ). (B) The right panel focuses on the gray colored midrange of the graph to demonstrate that most of the motor improvement occurs in the first half of the training for those at the 50\% level of F-M score (a patient with a moderate severity stroke), while the improvement occurs throughout the entire training period for those at the $75 \%$ level of the F-M score (a patient with a mild to moderate stroke). Also there is further improvement after the training has ended for those with less severe initial injury (and higher F-M scale scores) (4). The panels are adapted from (4): Bruce T Volpe, Patricio T Huerta, Johanna L Zipse, Avrielle Rykman, Dylan Edwards, Laura Dipietro, Neville Hogan, Hermano I Krebs. Robotic Devices as Therapeutic and Diagnostic Tools for Stroke Recovery. Arch. Neurol. 2009;66:1086-90. See http://archneur.jamanetwork.com/article.aspx?articleid=797921.

The clinical improvement for three of the trained groups was significant. The differences suggested that training can be individualized for a particular patient impairment.

\section{CAN EXPLICIT TRAINING OF THE AFFECTED LIMB GENERALIZE TO IMPROVEMENT ON UNTRAINED MOTOR TASKS?}

It is a standard procedure that all patients prior to robot training are asked to draw a circle by holding onto the end of the robot and using it to draw a circle. The typical attempt appears in Figure 3. In a trial of 117 patients who were being treated for chronic stroke with 9 wks of robotic therapy, we were able to analyze the circle drawing by measuring the axes of the circle before and after robot treatment and also by measuring the elbow flexion and extension and the shoulder adduction and abduction (15). The data show that patients improve their circle figures by coordinating their elbow and shoulder in a new way so that the major and minor axes of what had been an ellipse approach unity and therefore the resultant figure is closer to a circle (Figure 4). Despite training on point-to-point tasks in which the performance stresses movement in straight lines, these data illustrate new and the groups were trained under three different robot-controller conditions: first, trajectory control (slot-guidance training); second, increased resistance to movement (strength training); third, standard conditions of getting out of the way when the patient moves (standard training). Table 1 demonstrates the change in clinically measured motor function difference (Fugl-Meyer scale scores) between admission and discharge. The robot-derived kinetic and kinematic measures for the patients in each training condition demonstrate training specificity. Namely, the group that was trained to improve trajectory had the best aiming scores and the group trained to improve strength demonstrated the highest force scores.
Table 1. Improved movement after robot therapy depends on the kinematic details of the robot training.

\begin{tabular}{llcc}
\hline & \multicolumn{3}{c}{ Controller tuned to: } \\
\cline { 2 - 4 } & Slot guidance & Strength & Standard \\
\hline Change: admission to discharge $^{\mathrm{a}}$ & $7.3(1.29)$ & $4.5(0.81)$ & $4.5(0.91)$ \\
Aiming, radian & $-0.162(0.035)^{\mathrm{b}}$ & $-0.048(0.045)$ & $-0.027(0.054)$ \\
Speed, second & $-0.628(0.669)$ & $-1.838(0.409)$ & $-3.542(1.189)^{\mathrm{b}}$ \\
Force, newton & $0.503(3.143)$ & $11.666(3.536)^{\mathrm{b}}$ & $3.959(3.826)$ \\
\hline
\end{tabular}

This table shows that the controller tuned to train different aspects of movement leads to differential outcome in 111 patients with hemiparesis after chronic stroke. The improvement in Fugl-Meyer (F-M) scale score is significant for each group with the slotguidance-trained group demonstrating greater improvement. The slot-guidance tuning also yielded significantly better aiming scores. Greater strength change occurred when the controller was tuned to increase the resistance. The standard program generated the significantly faster performance.

${ }^{a}$ Change is indicated as difference from admission to discharge as measured by the F-M scale score $(P<0.05)$.

${ }^{\mathrm{b}} P<0.01$. 
$3 \mathrm{~A}$

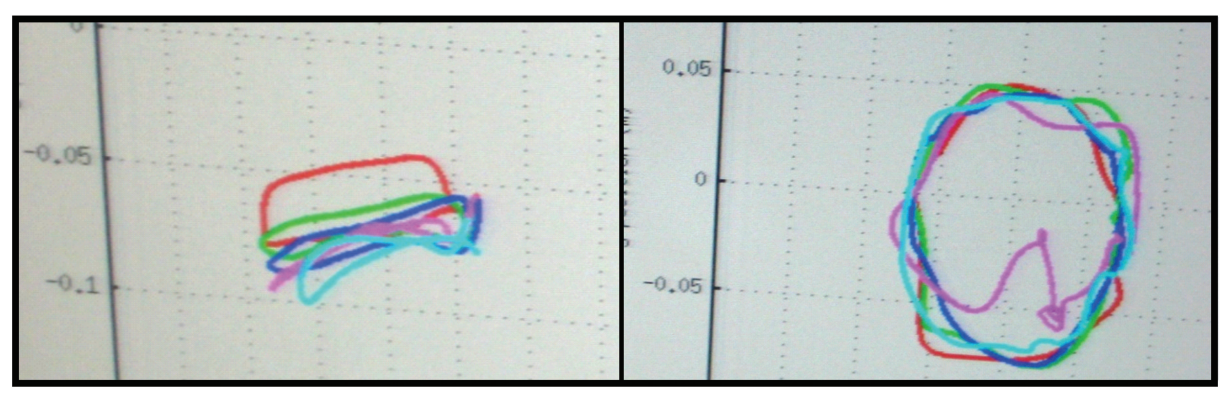

Figure 3. (A) This panel shows a screen shot of the drawing of "the best circle" that a patient executed with the robot at the beginning of the training trial. (B) This panel shows a screen shot of the drawing of "the best circle" that the same patient drew at the end of the training trial.

control of the joint angles of the elbow and shoulder. Specifically the elbow and shoulder become less correlated, as in normal, because control improves and spasticity and synergy decrease.

The data show that motor performance may generalize beyond the trained tasks. The clinically significant changes support patient anecdotes of being able to use eating utensils more effectively, being able to bath and dress independently, and, of course, there is always a sportsperson, in our case, an avid golfer, who returned to the links.

\section{CAN THE CONTROL GROUP BE TRAINED IN AN INTENSIVE MANNER TO MIMIC THE ROBOT TRAINING?}

Training intensity has been at the forefront of many therapy programs in restorative neurology for years (16-19), and recently there is discussion about the timing of intensive movement therapies $(20,21)$. There is little doubt that intensive movement-based therapist-delivered programs will improve outcome, the issue is that the difficult physical work is not reasonable to ask of a therapist for every patient all day long. Frankly, the therapist has other skills to aid and abet recovery. And this human factor is among the strong points of support for an interactive robot training program. In our study, we randomized 57 patients with chronic stroke to be treated with an intensive movement protocol delivered by robot or therapist. The two treatment groups had comparable and significant improvement on motor impairment scales (Figure 5) (22). Many have asked for the protocol because it has been used successfully, most recently in a multicenter trial of robotic training as a positive control (7).

\section{$4 \mathrm{~A}$}

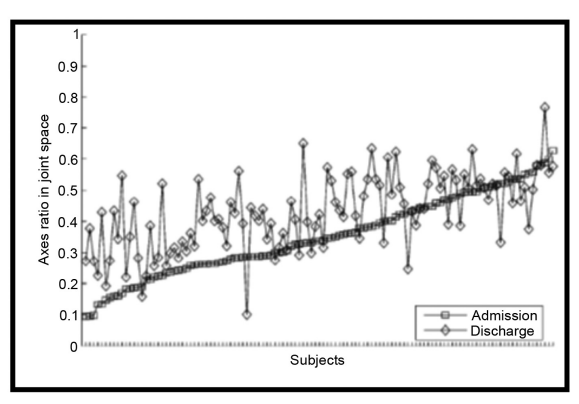

Essentially intensive motor training improves outcome for the paretic limb, and whether this activity is delivered by a therapist or a robot appears to make little difference, except to the therapist.

\section{CAN THE ROBOT-DERIVED MEASUREMENTS OF MOVEMENT PROVIDE AN ACCURATE OBJECTIVE RELEVANT BIOMARKER?}

Much as robotic devices are critical adjuncts to deliver intensive treatment in stroke recovery trials, clinical research protocols in stroke, especially for drug outcome studies, would be aided by a reliable, repeatable and temporally efficient continuous measure of impairment. Recently we were able to measure the movement of the paralyzed upper limb in a longitudinal manner in 208 patients who were recovering from stroke (23). We tested the relevance of the relationship among four standard and well-known clinical movement impairment scales and objective robot measures. For the clinical

\section{B}

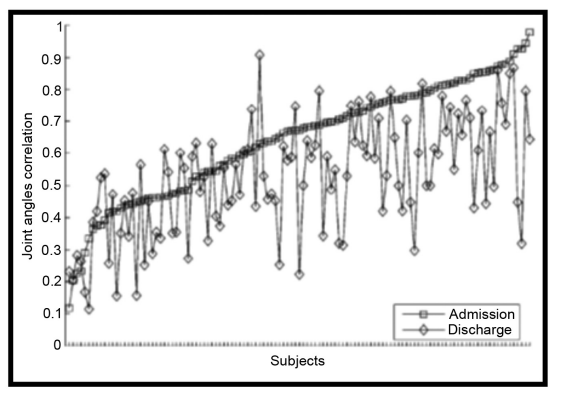

Figure 4. (A) This panel quantifies the circle drawing task by measuring the length and ratio of the major and minor axes of the first drawing (as in Figure $3 A$ for example) which is an ellipse and the drawing after training in which the ratio approaches unity as the patient can now coordinate and control the elbow and shoulder and can draw a recognizable circle. The tightly packed squares represent the ratio of the initial circle drawn by each of 117 patients with chronic stroke. The diamonds, almost all above the line of admission squares, represent the ratio of the axes for the circles that the patients drew at discharge. The drawings are significantly improved $(N=117 ; p<0.05)$. (B) This panel demonstrates the increased independent control of elbow or the shoulder as a decrease in the correlation of the movement of the joints. The line of squares indicates the admission correlation of shoulder and elbow movement for the same 117 patients with chronic stroke. Normal correlation values are around 0.2 (unpublished results). The diamonds represent the discharge correlation and for most patients there is a return toward normal values $(N=117 ; p<0.05)$ (15). The panels are adapted from (15): L Dipietro, HI Krebs, SE Fasoli, BT Volpe, J Stein, C Bever, N Hogan. Changing Motor Synergies in Chronic Stroke. J. Neurophysiol. 2007:98:757-68. See http://jn.physiology.org/content/98/2/757.long. 


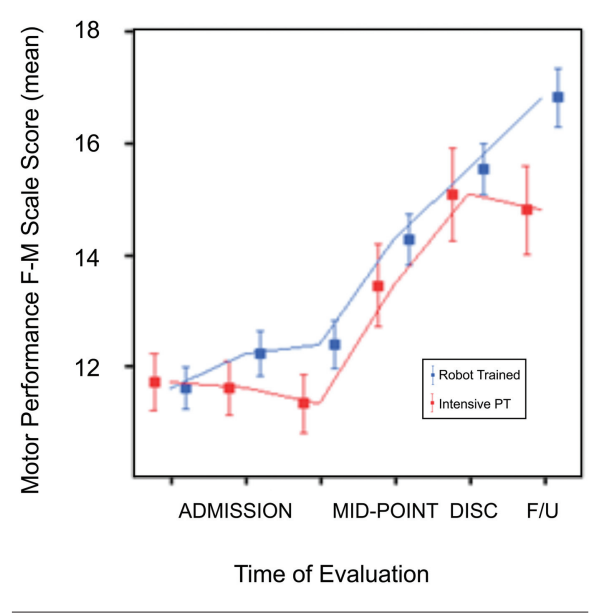

Figure 5. This figure demonstrates the motor power as measured by the Fugl-Meyer (F-M) scale in patients with chronic stroke treated with robotic training $(n=47)$ and intensive therapist training $(n=10)$. The performance of patients with stroke treated with either technique is comparable on admission (represented by three measures taken one week apart); midpoint; and discharge (DISC). However, the robotic-trained patients appear to continue to improve after the end of training (22). The graph is based on information from (22): Bruce T Volpe, Daniel Lynch, Avrielle RykmanBerland, Mark Ferraro, Michael Galgano, Neville Hogan, and Hermano I Krebs. Intensive Sensorimotor Arm Training Mediated by Therapist or Robot Improves Hemiparesis in Patients With Chronic Stroke. Neurorehabil. Neural Repair. 2008;22:305-10. See http://nnr.sagepub.com/content/22/3/305.refs. F/U, follow-up.

scales, therapists were trained and interand intrarater reliability was assured (24). For the robot measure we used a series of visually guided and visually evoked unconstrained reaching and attempts to move against resistance (provided by the robot) (25). From these movements, we were able to calculate deviation from a straight line, aiming, average, peak speed and duration of movement, and also characterize the movement smoothness. The robotic measures predicted well the clinical measures, so for the standard FuglMeyer impairment assessment scale the $R^{2}=0.73$, and for the standard Motor Power scale the $R^{2}=0.75$. These results suggest that the robot measures of motor performance adequately capture outcome and make the robotic device a useful tool that provides objective, repetitive, reliable and speedily obtained measurements of motor performance.

\section{CAN THE ROBOT TRAINING EMERGE AS A PLATFORM FOR COMBINATION THERAPY?}

Recently we demonstrated that the use of transcranial direct current stimulation (tDCS) could alter the performance on the robot in 12 patients with chronic stroke (26). For this study, a $2 \mathrm{~mA}$ current was delivered by a battery-driven stimulator for 20 min before, during or after robotmeasured movement tasks. Standard procedures with transcranial magnetic stimulation (TMS) were used to determine the optimal site for the anode and for the sham control procedure. The cathode was placed on the contralateral superorbital region. While the data showed that 20 min of $\mathrm{tDCS}$ before but not during or after robot-measured movement performance led to significant improvement in smoothness, tDCS during robotic measure interfered with aiming, and tDCS after robot measure significantly decreased mean speed (Figure 6). These data support the effectiveness of the robot used in conjunction with other noninvasive technology to improve recovery. Further the data show that noninvasive treatments need to be properly investigated as timing of the treatment with respect to robotic training may render deleterious effects.

\section{DISCUSSION}

Real world rehabilitation techniques have been influenced by preclinical experiments and clinical observations that have demonstrated the felicitous effects of "intensive" motor training before the dawn of the "electroceutical" revolution in robotics (27-30). Similarly, clinical investigators have produced important trial information to show that dedicated stroke units where therapists deliver intensive movement programs provide the best outcome $(20,31,32)$. And, finally, these experimental findings are in accord with the detailed anecdotal reports of particular patients who have docu-

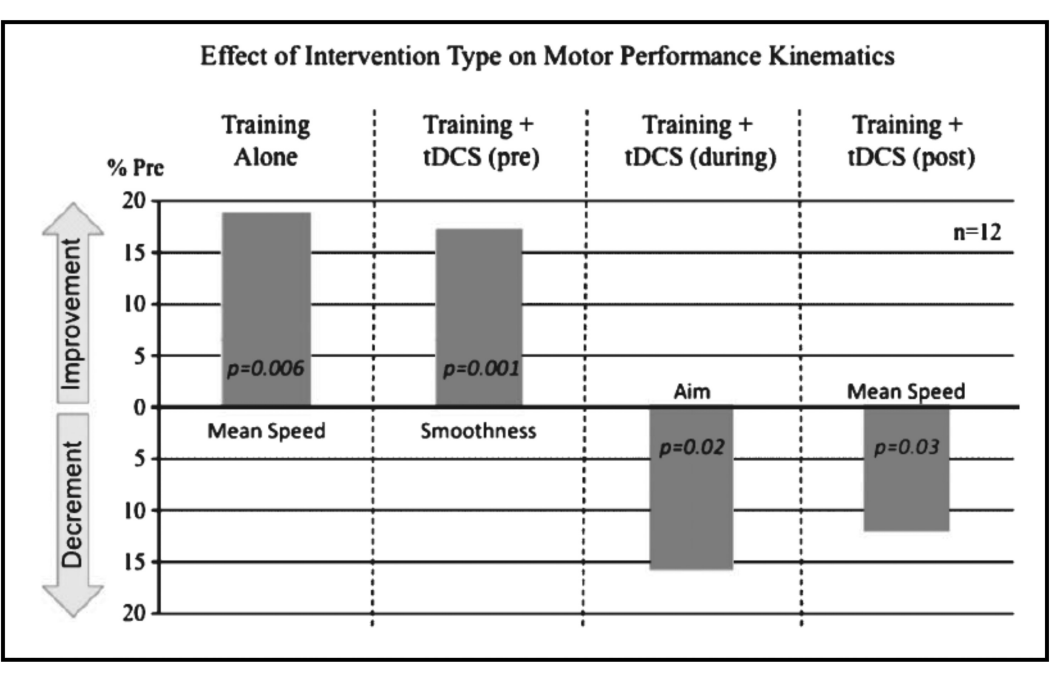

Figure 6. This image demonstrates in 12 patients with chronic stroke that the timing of tDCS improves smoothness of movement when applied before robot treatment, yet interferes with aiming and mean speed when applied during or after robot training. These effects are significant (26). The image is adapted from (26): $\bigvee$ Giacobbe, HI Krebs, BT Volpe, A Pascual-Leone, A Rykman, G Zeiarati, F Fregni, L Dipietro, GW Thickbroom, DJ Edwards. Transcranial direct current stimulation (tDCS) and robotic practice in chronic stroke: The dimension of timing. NeuroRehabilitation. 2013;33:49-56. See http://iospress.metapress.com/content/x219150u616h1078/. 
mented extraordinary motor recovery after stroke that appeared to depend on effort and intensive practice (33-35).

Stroke is the number one cause of permanent disability (36). And this dubious leadership position is unlikely to recede because surprisingly high incidence information for stroke is driven by an aging population and better epidemiology from urban populations, and recent equally surprising prevalence information is driven by improved survival after the acute event, and also the emerging use of the only effective treatment for acute stroke, tissue plasminogen activator.

\section{CONCLUSION}

The conclusions regarding the problem of treating persistent permanent neurological impairment has to focus on new technology. Since the answer to each argument presented here is, resoundingly, yes, it behooves rehabilitation enterprises to adopt robotic devices as important adjuncts to the current therapy protocols. Moreover, as the science of clinical recovery proceeds to be measured by waves of small molecules, or novel drugs, or cellbased therapies that repair or alter central nervous system networks, then the ultimate phenotypic motor reorganization will require retraining. With robotics, therapists are finally armed with tools that will deliver reproducible, intensity controlled retraining alone, or in combination with other invasive or noninvasive brain stimulation. And finally, these robotic devices will provide objective measurements for any study of motor recovery.

\section{ACKNOWLEDGMENTS}

BT Volpe was supported from NIH RO1-HD069776, the Buchanan Family Foundation and the Feinstein Institute for Medical Research.

\section{DISCLOSURE}

The authors declare that they have no competing interests as defined by Bioelectronic Medicine, or other interests that might be perceived to influence the results and discussion reported in this paper.

\section{REFERENCES}

1. Aisen ML, Krebs HI, Hogan N, McDowell F, Volpe BT. (1997) The effect of robot-assisted therapy and rehabilitative training on motor recovery following stroke. Arch. Neurol, 54:443-6.

2. Hogan N, et al. (2006) Motions or muscles? Some behavioral factors underlying robotic assistance of motor recovery. J. Rehabil. Res. Dev. 43:605-18.

3. Krebs HI, Volpe BT. (2013) Rehabilitation robotics. Handb. Clin. Neurol, 110:283-94.

4. Volpe BT, et al. (2009) Robotic devices as therapeutic and diagnostic tools for stroke recovery. Arch. Neurol. 66:1086-90.

5. Ferraro M, et al. (2003) Robot-aided sensorimotor arm training improves outcome in patients with chronic stroke. Neurology. 61:1604-7.

6. Krebs HI, et al. (2003) Rehabilitation robotics: performance-based progressive robot-assisted therapy. Autonomous Robots. 15:7-20

7. Lo AC, et al. (2010) Robot-assisted therapy for long-term upper-limb impairment after stroke. N. Engl. J. Med. 362:1772-83.

8. Miller EL, et al. (2010). Comprehensive overview of nursing and interdisciplinary rehabilitation care of the stroke patient: a scientific statement from the American Heart Association. Stroke. 41:2402-48.

9. Volpe BT, Krebs HI, Hogan N, Edelstein OL, Diels C, Aisen M. (2000) A novel approach to stroke rehabilitation: robot-aided sensorimotor stimulation. Neurology. 54:1938-44.

10. Page SJ, Gater DR, Bach YRP. (2004) Reconsidering the motor recovery plateau in stroke rehabilitation. Arch. Phys. Med. Rehabil. 85:1377-81.

11. Dobkin BH. (2008) Training and exercise to drive poststroke recovery. Nat. Clin. Pract. Neurol. 4:76-85.

12. Krebs HI. (1997) Robot-aided neuro-rehabilitation and functional imaging [dissertation]. Cambridge (MA): Massachusetts Institute of Technology.

13. Fugl-Meyer AR, Jaasko L, Leyman I, Olsson S, Steglind S. (1975) The post-stroke hemiplegic patient. 1. a method for evaluation of physical performance. Scand. J. Rehabil. Med. 7:13-31.

14. Palazzolo JJ. (2005) Robotic technology to aid and assess recovery and learning in stroke patients [dissertation]. Cambridge (MA): Massachusetts Institute of Technology.

15. Dipietro L, et al. (2007) Changing motor synergies in chronic stroke. J. Neurophysiol. 98:757-68.

16. Butefisch C, Hummelsheim H, Denzler P, Mauritz KH. (1995) Repetitive training of isolated movements improves the outcome of motor rehabilitation of the centrally paretic hand. J. Neurol. Sci. 130:59-68.

17. Feys HM, et al. (1998) Effect of a therapeutic intervention for the hemiplegic upper limb in the acute phase after stroke: a single-blind, randomized, controlled multicenter trial. Stroke. 29:785-92.

18. Kwakkel G, Wagenaar RC, Twisk JW, Lankhorst GJ, Koetsier JC. (1999) Intensity of leg and arm training after primary middle-cerebral-artery stroke: a randomised trial. Lancet. 354:191-6.

19. Whitall J, McCombe Waller S, Silver KH, Macko RF. (2000) Repetitive bilateral arm training with rhythmic auditory cueing improves motor function in chronic hemiparetic stroke. Stroke. 31:2390-5.

20. Breceda EY, Dromerick AW. (2013) Motor rehabili- tation in stroke and traumatic brain injury: stimulating and intense. Curr. Opin. Neurol. 26:595-601.

21. Dromerick AW, et al. (2009) Very early constraintinduced movement during stroke rehabilitation (VECTORS): A single-center RCT. Neurology. 73:195-201.

22. Volpe BT, et al. (2008) Intensive sensorimotor arm training mediated by therapist or robot improves hemiparesis in patients with chronic stroke. Neurorehabil. Neural Repair. 22:305-10.

23. Krebs HI, et al. (2014) Robotic measurement of arm movements after stroke establishes biomarkers of motor recovery. Stroke. 45:200-4.

24. Ferraro M, et al. (2002) Assessing the motor status score: a scale for the evaluation of upper limb motor outcomes in patients after stroke. Neurorehabil. Neural Repair. 16:283-9.

25. Bosecker C, Dipietro L, Volpe B, Krebs HI. (2010) Kinematic robot-based evaluation scales and clinical counterparts to measure upper limb motor performance in patients with chronic stroke. Neurorehabil. Neural Repair. 24:62-9.

26. Giacobbe V, et al. (2013) Transcranial direct current stimulation (tDCS) and robotic practice in chronic stroke: the dimension of timing. NeuroRehabilitation. 33:49-56.

27. Luft AR, et al. (2004) Repetitive bilateral arm training and motor cortex activation in chronic stroke: a randomized controlled trial. JAMA. 292:1853-61.

28. Nudo RJ, Milliken GW, Jenkins WM, Merzenich MM. (1996) Use-dependent alterations of movement representations in primary motor cortex of adult squirrel monkeys. J. Neurosci. 16:785-807.

29. Nudo RJ, Wise BM, SiFuentes F, Milliken GW. (1996) Neural substrates for the effects of rehabilitative training on motor recovery after ischemic infarct. Science. 272:1791-4.

30. Pascual-Leone A, Nguyet AD, Cohen LG, BrasilNeto JP, Cammarota A, Hallett M. (1995) Modulation of muscle responses evoked by transcranial magnetic stimulation during the acquisition of new fine motor skills. J. Neurophysiol. 74:1037-45.

31. Indredavik B, Bakke F, Slordahl SA, Rokseth R, Haheim LL. (1999) Stroke unit treatment. 10-year follow-up. Stroke. 30:1524-7.

32. Jorgensen HS, et al. (2000) Who benefits from treatment and rehabilitation in a stroke Unit? A community-based study. Stroke. 31:434-9.

33. Aguilar MJ. (1969) Recovery of motor function after unilateral infarction of the basis pontis. Report of a case. Am. J. Phys. Med. 48:279-88.

34. Bach YRP. (2005) Emerging concepts of brain function. J. Integr. Neurosci. 4:183-205.

35. Brodal A. (1973) Self-observations and neuroanatomical considerations after a stroke. Brain. 96:675-94.

36. Go AS, et al. (2014) Heart disease and stroke statistics-2014 update: a report from the American Heart Association. Circulation. 129:e28-292.

Cite this article as: Volpe BT. (2014) Bioelectronic medicine and the dawn of robotic training to improve motor outcome in chronic stroke. Bioelectron. Med. 1:9-14 